\title{
Un Caso de la Modelización de Series de Tiempo Bivariada para Determinar el Orden de los Hiperparámetros en el Modelo Estructural
}

Rosa Medina ${ }^{1}$

(Recibido: 30/03/2016

\author{
Ana Cárdenas ${ }^{2}$
}

Aceptado: 21/04/2016)

Resumen: Se busca formular modelos que expliquen la captura - desembarque de anchoveta en función de periodos fríos y cálidos en el mar peruano frente a las costas del Callao. Se realiza un estudio estadístico de las características de la temperatura superficial del mar específicamente con las componentes observables y no observables de ésta, que pueden estar asociadas con los eventos conocidos como "El Niño " y "La Niña". Se desarrolla modelos estructurales para estimar, con ayuda del filtro de Kalman, el comportamiento de la captura desembarque de anchoveta en función de la temperatura superficial del mar. Se formula un modelo estructural mediante la metodología espacio-estado que incorpora los conceptos de series de tiempo bivariadas por enfoque espectral.

Palabras Claves: Modelo estructural, espacio-estado, Filtro de Kalman, espectro cruzado, coherencia espectral, Evento "El Niño", desembarque de anchoveta.

\section{A Case of Bivariate Time Series Modeling to Determine the Order of the Hyperparameters in Structural Model}

\begin{abstract}
It seeks to develop models that explain the catch-landing of anchovy in terms of cold and warm periods in the Peruvian sea off the coast of Callao. A statistical study is performed, of the characteristics of the sea surface temperature specifically with observable and unobservable components thereof, which may be associated with the events known as "El Niño"and "La Niña ".

It develops structural models to estimate, using the Kalman filter, the behavior of the landing anchovy catch depending on the surface temperature of the sea. A structural model is formulated using the state-space methodology that incorporates the concepts of time series by bivariate spectral approach.
\end{abstract}

Key Words: Structural model, space-state, Kalman filter, cross spectrum, spectral coherence, Event El "El Niño", landing of anchovy.

\section{Introducción}

En la presente investigación aplicada se presenta el caso desembarque de Anchoveta y la temperatura superficial del mar frente a las costas del Callao. Según la Dirección de Estudios Pelágicos - IMARPE, el volumen de desembarque de anchoveta en el Callao es el segundo más alto de la región norte-centro, superado solo por Pisco. Esto justifica porque se ha elegido como lugar de investigación este puerto.

La distribución de la anchoveta está fuertemente asociada a la extensión de las aguas costeras frías, caracterizadas por sus bajas temperaturas además otros factores ambientales.

\footnotetext{
${ }^{1}$ UNMSM, Facultad de Ciencias Matemáticas, e-mail:rmedinam@unmsm.edu.pe

${ }^{2}$ UNMSM, Facultad de Ciencias Matemática, e-mail: acardenasr@unmsm.edu.pe
} 
Las investigaciones de IMARPE hacen posible concluir que las condiciones oceanográficas frente a las costas peruanas en los últimos años representan tendencia hacia una mayor variabilidad intra-anual en el régimen térmico hacia una estructura vertical más profundizada que desfavorece el transporte de nutrientes a la superficie. Para finales del 2014 se esperaba que entre noviembre y diciembre se presentaran condiciones anómalas en cuanto a temperatura las cuales afectarían el ecosistema de afloramiento peruano. Por otro lado, los modelos internacionales de predicción del clima pronosticaron una mayor probabilidad de desarrollo de condiciones de El Niño hasta marzo del 2015, sin conocer la magnitud que tendría.

Se formula las siguientes preguntas:

- ¿Cuáles son las características de las componentes periódicas de la temperatura superficial del mar peruano, específicamente frente a las costas del Callao?

- ¿Cómo influye la temperatura superficial en el comportamiento de la anchoveta frente a las costas del Callao?

- ¿Qué factores de la temperatura superficial del mar explican el comportamiento de la anchoveta?

- ¿De qué manera la modelización de series de tiempo bivariada determinará el orden de los hiperparámetros en el modelo estructural?

La gran biomasa de anchoveta originó, a partir de mediados de la década de 1950, una gran industria de harina y aceite de pescado, con el crecimiento más espectacular a nivel mundial que llevó al Perú, a partir de 1959, a constituirse en el mayor productor de harina y aceite de pescado en el mundo.

Sin embargo la anchoveta es un recurso pelágico, muy sensible a los cambios ambientales entre los que podemos citar a la temperatura superficial del mar. En consecuencia las alteraciones ocasionadas por las variaciones de El Niño y de La Niña han causado importantes cambios en su ecosistema y hábitat.

Estudios realizados en IMARPE, dan cuenta de la preocupación de científicos marinos (biólogos, oceanógrafos, químicos, físicos, ingenieros, etc.) y del sector económico, por las alteraciones que las variaciones en la temperatura superficial del mar ocasiona sobre los principales recursos del mar, dentro de los que destaca la anchoveta. IMARPE ha desarrollado eventos y publicaciones referentes a este tema y producto de su preocupación se convocaron consultores para el desarrollo de cursos y consultorías sobre el análisis de series de tiempo aplicada a la temperatura superficial del mar y a la biomasa de la anchoveta.

Desde el punto de vista estadístico la modelización de series de tiempo mediante los modelos SARIMA, requiere la determinación de la longitud de la periodicidad, la misma que es estática, razón por la cual se presentan pobres estimaciones en series de tiempo estacionales o de lo contrario obliga a incluir métodos como estacionalidad múltiple, filtros estacionales parciales, etc.

El objetivo de éste artículo es caracterizar la temperatura superficial del mar y su influencia en el desembarque de anchoveta frente a las costas del Callao, mediante modelos estructurales de series de tiempo y ver de qué manera la modelización de series de tiempo bivariada ayudará a determinar el orden de los hiperparámetros en dichos modelos.

La metodología empleada corresponde a los modelos estructurales en el tiempo que son desarrollados en base a los modelos estado-espacial usando los filtros de Kalman y con apoyo de otras técnicas como la modelización por series bivariadas para la estimación de los hiperparámetros de los modelos estructurales.

Por ello se propone el desarrollo de series de tiempo estructurales como una metodología para la estimación y análisis de la Biomasa de Anchoveta medida mediante el volumen de captura 
en función de la Temperatura superficial del Mar, ambas series frente a las costas del Callao. Estudiaremos las características de esta asociación en términos de su periodicidad, la cual se sospecha que es variable, y que ocasionan el calentamiento y enfriamiento de nuestras aguas con severas consecuencias sobre los principales recursos hidrobiológicos del mar peruano.

Sabiendo que hasta el momento los biólogos marinos consideran que el calentamiento-enfriamiento de las aguas corresponde a ciclos de longitud variable, creemos que al analizar las características cíclicas de la temperatura superficial del mar, podremos conocer sus repercusiones en los recursos pelágicos específicamente en la anchoveta.

También creemos posible construir un modelo que incorpore esta asociación y permita identificar la variación de esta periodicidad con la finalidad de que los expertos en estudios marinos cuenten con una herramienta científica que les permita identificar los factores asociados a la biomasa y temperatura no evidenciados a simple vista, así como su variabilidad. De esta manera podrán tomar las previsiones necesarias para evitar la pesca de elementos juveniles de esta especie que provocaría una ruptura en la cadena marina.

En la sección 2, se presenta la metodología de Modelos Estructurales de series de tiempo y en la sección 3 se presenta los resultados y la discusión de la aplicación del método con apoyo de otras técnicas como la modelización por series bivariadas.

\section{Metodología}

\subsection{Modelos estructurales para series de tiempo univariadas}

Partiendo del modelo básico lineal general estructural (Douc, Moulines, \& Stoffer, 2014):

$$
\left.\begin{array}{ll}
\text { Ecuación de observación } & Z_{t}=A_{t} X_{t}+w_{t} \\
\text { Ecuación de estado } & X_{t}=G_{t} X_{t-1}+v_{t}
\end{array}\right\}
$$

donde:

$$
\begin{array}{ll}
Z_{t}: & \text { vector de observación } \\
A_{t}: & \text { matriz de sistema }(q \times p) \\
X_{t}: & \text { proceso de markov de dimensión } p \\
w_{t}: & \text { vector ruido }(q \times 1) \\
G_{t}: & \text { matriz transición }(p \times p) \\
v_{t}: & \text { vector ruido }(p \times 1)
\end{array}
$$

Se tienen las siguientes propiedades:

$$
\begin{array}{lll}
\text { i. } & E\left(v_{t} v_{t+k}\right)=0 \\
\text { ii. } & E\left(v_{t}\right)=0 & \\
\text { iii. } & \operatorname{Cov}\left(v_{t}\right)=Q \quad \operatorname{Cov}\left(w_{s}\right)=R \\
\text { iv. } & E\left(v_{t} w_{s}^{\prime}\right)=0 \quad \text { para todo } t, s=1,2, \ldots, N \\
\text { v. } & E\left(v_{t} x_{0}^{\prime}\right)=0 \quad E\left(w_{t} x_{0}^{\prime}\right)=0 \\
\text { vi. } & \text { Si } v_{t} \text { y } w_{t} \text { se distribuyen en forma normal, entonces el modelo es gaussiano } \\
\text { vii. } & \text { El modelo univariado corresponde a } q=1
\end{array}
$$

Dentro de un marco de regresión, una tendencia simple sería modelada en términos de una constante por el tiempo con un disturbio, o error aleatorio, aditivo, es decir:

$$
Y_{t}=\alpha+\beta_{t}+\epsilon_{t}, t=1, \ldots, N
$$

Este modelo es fácil de estimar usando mínimos cuadrados simple, pero tiene la desventaja de considerar la tendencia como una componente determinística. Permitiendo que los coeficientes 
$\alpha$ y $\beta$ sean dinámicos, como procesos estocásticos, se introduce flexibilidad al modelo y por lo tanto la tendencia se puede adaptar a los cambios que se presenten.

La estimación actual o filtrada de la tendencia se consigue escribiendo el modelo en su forma de espacio estado tal como en (1) y aplicando luego el fitro de Kalman. El filtrado implica calcular el mejor estimador en cada momento t usando las observaciones.

Los parámetros de los modelos espacio estado reciben el nombre de hiperparámetros, de esta forma se diferencian de los elementos del vector de estado.

La magnitud por la cual los parámetros aleatorios pueden variar depende de los hiperparámetros, los que pueden estimarse por máximo verosimilitud.

\section{Enfoque de dominio del Tiempo}

Este enfoque parte del supuesto de que un modelo general para cualquier serie estacionaria no determinística puede ser representada como un modelo autorregresivo de media móvil $\operatorname{ARMA}(\mathrm{p}, \mathrm{q})$. Identifica el orden del modelo, es decir determina los valores de $p$ y $q$ luego de inspeccionar el correlograma estimado y el correlograma parcial estimado.

Se estima el modelo, bajo el supuesto de normalidad de los errores. Posteriormente se analizan los residuos los cuales deben presentar un comportamiento aleatorio, para ello se realizan dócimas con estadísticos de prueba como la prueba de Box y Pierce o la prueba de Box y Lung, las cuales se usan las primeras $k$ autocorrelaciones residuales. Si la prueba es no significativa el modelo es satisfactorio y el modelo puede ser usado para predecir, de lo contrario se debe regresar a identificar otro modelo, evitando la sobre parametrización, es decir tratando que $p$ y $q$ sean pequeños (Box, Jenkins, \& Reinsel, 1994).

En los casos en los cuales las series no son estacionarias se aplican diferencias finitas de orden "d" y se procede con los pasos de identificación, modelización, verificación y pronósticos. Así mismo los efectos estacionales también pueden ser incorporados mediante modelización SARIMA $(p, d, q)$, $(P, D ; Q)$ (Box, Jenkins, \& Reinsel, 1994).

La metodología de selección de modelos para los modelos estructurales pone menos énfasis en la observación del correlograma de la serie de tiempo y más bien se pone mayor atención a la formulación del modelo en términos de componentes cuya presencia se sugieren a partir del conocimiento preliminar del fenómeno bajo estudio y de la inspección del gráfico de la serie original. Así por ejemplo para la serie desembarque mensual de anchoveta, debería incorporarse la componente estacional desde el comienzo $y_{t}=\mu_{t}+\gamma_{t}+\epsilon_{t}, t=1, \ldots, n$ y se extraerá sólo si se verifica que es no significativa.

Considérese la componente tendencia lineal representada por $\beta_{t}$ y la componente estacional $\gamma_{t}$. Esta componente define su longitud estacional $(s)$, dependiendo de la periodicidad conceptual de la serie en el año. De esta forma $s=12$ para estacionalidad mensual en un año, $s=4$ para datos trimestrales, $s=365$ para datos diarios, siendo posible que $s$ tome otros valores dependiendo de la definición de su estacionalidad.

Otra forma de representación es mediante la representación ARIMA. Definiendo $r=\max (p, q+$ 1)

$$
\begin{gathered}
Y_{t+1}-\mu=\phi_{1}\left(Y_{t}-\mu\right)+\phi_{2}\left(Y_{t-1}-\mu\right)+\cdots+\phi_{r}\left(Y_{t-r}-\mu\right)-a_{t} \\
+\theta_{1} a_{t-1}+\theta_{2} a_{t-2}+\cdots+\theta_{r-1} a_{t-r+1}
\end{gathered}
$$

donde: $\phi_{j}=0, \forall j>p$ y $\theta_{j}=0, \forall j>p$. 
Ecuación de Estado $(r=\max (p, q+1))$ :

$$
\xi_{t+1}=\left(\begin{array}{ccccc}
\phi_{1} & \phi_{2} & \cdots & \phi_{r-1} & \phi_{r} \\
1 & 0 & \cdots & 0 & 0 \\
0 & 1 & \cdots & 0 & 0 \\
\vdots & \vdots & \ddots & \vdots & \vdots \\
0 & 0 & \cdots & 1 & 0
\end{array}\right)_{r \times r} \xi_{r}+\left(\begin{array}{c}
a_{r+1} \\
0 \\
0 \\
\vdots \\
0
\end{array}\right)_{r \times 1}
$$

Ecuación de Observación $(n=1)$ :

$$
Y_{t}=\mu+\left(\begin{array}{ccccc}
1 & \theta_{1} & \theta_{2} & \cdots & \theta_{r-1}
\end{array}\right)_{1 \times r} a_{t}
$$

Para el caso de la captura-desembarque de anchoveta, consideremos un escalar $C_{t}$ no observable que representa el estado del ciclo climático. Un conjunto de $n$ observaciones biológicas $\left(Y_{1 t}, Y_{2 t}, \ldots, Y_{n t}\right)$ se asumen influidas por el ciclo climático y además una componente $X_{i t}$ correlacionado con $Y_{j t}, \forall i \neq j$. Si el ciclo climático puede ser descrito como un proceso $A R(1)$, entonces el vector estado $((n+1) \times 1)$ puede ser:

$$
\xi_{t}=\left(\begin{array}{c}
C_{t} \\
X_{1 t} \\
X_{2 t} \\
\vdots \\
X_{n t}
\end{array}\right)
$$

con ecuación de estado

$$
\left(\begin{array}{c}
C_{t+1} \\
X_{1 t+1} \\
X_{2 t+1} \\
\vdots \\
X_{n t+1}
\end{array}\right)_{(n+1) \times 1}=\left(\begin{array}{ccccc}
\phi_{c} & 0 & 0 & \cdots & 0 \\
0 & \phi_{1} & 0 & \cdots & 0 \\
0 & 0 & \phi_{2} & \cdots & 0 \\
\vdots & \vdots & \vdots & \ddots & \vdots \\
0 & 0 & 0 & \cdots & \phi_{n}
\end{array}\right)_{(n+1) \times(n+1)}\left(\begin{array}{c}
C_{t} \\
X_{1 t} \\
X_{2 t} \\
\vdots \\
X_{n t}
\end{array}\right)_{(n+1) \times 1}+\left(\begin{array}{c}
V_{c t+1} \\
V_{1 t+1} \\
V_{2 t+1} \\
\vdots \\
V_{n t+1}
\end{array}\right)_{(n+1) \times 1}
$$

Ecuación de observación:

$$
\left(\begin{array}{c}
Y_{1 t} \\
Y_{2 t} \\
\vdots \\
X_{n t}
\end{array}\right)_{n \times 1}=\left(\begin{array}{c}
\mu_{1} \\
\mu_{2} \\
\vdots \\
\mu_{n}
\end{array}\right)_{n \times 1}+\left(\begin{array}{ccccc}
\gamma_{1} & 1 & 0 & \cdots & 0 \\
\gamma_{2} & 0 & 1 & \cdots & 0 \\
\vdots & \vdots & \vdots & \ddots & \vdots \\
\gamma_{n} & 0 & 0 & \cdots & 1
\end{array}\right)_{n \times n}\left(\begin{array}{c}
C_{t} \\
X_{1 t} \\
\vdots \\
X_{n t}
\end{array}\right)_{n \times 1}+\left(\begin{array}{c}
W_{c, t+1} \\
W_{1, t+1} \\
\vdots \\
W_{n, t+1}
\end{array}\right)_{(n+1) \times 1}
$$

Así $\gamma_{i}$ es un parámetro que describe la sensibilidad de la i-ésima serie de los ciclos climáticos. Para hacerlo dinámico de orden $p$, se reemplaza $C$ y $X_{i} t$ por el vector de orden $(p \times 1)$ : $\left(C_{t}, C_{t-1}, \ldots, C_{t-p+1}\right)^{\prime}$ y $\left(C_{i, t}, C_{i, t-1}, \ldots, C_{i, t-p+1}\right)^{\prime}$, tal que $\xi_{t}$ es un vector $(n+1) p \times 1$. Los escalares $\phi$ se reemplazan por matrices $F_{i}$ de orden $(p \times p)$ y bloques de cero aumentados en $H^{\prime}$ en la ecuación de orden $(n \times(p \times 1))$.

\section{Modelo de Regresión Lineal}

A partir de los modelos estructurales un modelo uniecuacional con variables exógenas se expresa como:

$$
Y_{t}=\mu_{t}+x_{t}^{\prime} \delta+\epsilon_{t}, \mu_{t}=\mu_{t-1}+\eta_{t}
$$


Si la varianza de la perturbación $\eta_{t}$ es cero se obtiene un modelo de regresión clásico. Cuando la varianza del término de perturbación $\eta_{t}$ es cero y la varianza de la perturbación $\eta_{t}$ es diferente de cero, entonces corresponde a una regresión en primeras diferencias.

Si ambas varianzas son diferentes de cero, se obtiene un modelo de función de transferencia, con el que puede representarse una relación bivariada para un proceso de entrada (temperatura) y un proceso de salida (desembarque de anchoveta). En general, para conocer el efecto de otras variables exógenas sobre la variable endógena de interés, las variables regresoras se añaden en la ecuación de observación del modelo. Dependiendo del tipo de modelo estructural, se tendrían situaciones diferentes. Si las variables de regresión se añaden a un modelo de nivel local, la ecuación de observación tomará la forma:

$$
Y_{t}=\mu_{t}+x_{j t}+\epsilon_{t} \sum_{j=1}^{k} \beta_{j t}
$$

donde $x_{j t}$ es un vector de variables predictoras continua y $\beta_{j t}$ es vector de coeficientes de regresión desconocidos para $j=1,2, \ldots, k$. Para el caso de una variable predictora con $\beta_{t}=\beta_{1 t}$, el modelo toma la forma $Y_{t}=\mu_{t}+\beta_{t} x_{t}+\epsilon_{t}, \epsilon_{t} \simeq N I D\left(0, \sigma_{\epsilon}^{2}\right)$ independientes con distribución normal.

$$
\begin{array}{ll}
\mu_{t+1}=\mu_{t}+\xi_{t} & \epsilon_{t} \simeq N I D\left(0, \sigma_{\xi}^{2}\right) \\
\beta_{t+1}=\beta_{t}+\gamma_{t} & \gamma_{t} \simeq N I D\left(0, \sigma_{\gamma}^{2}\right)
\end{array}
$$

para $t=1, \ldots, n$.

El modelamiento con $k$ variables exógenas implica $k$ ecuaciones de estado adicionales (Commandeur \& Koopman, 2007) El término de perturbación $\gamma_{t}$ para la componente de regresión usualmente se fija en cero para estabilizar la relación entre $y_{t}$ y $x_{t}$ para toda $t$. Sin embargo si se requiere una componente de regresión estocástica, puede introducirse en el modelo. Luego de estimar el modelo estructural se usa el mismo tipo de prueba de diagnóstico que en los modelos ARIMA (Box y Pierce, Box y Lung, etc). Para el caso del estadístico de Box y Lung sus grados de libertad son igual al número de autocorrelaciones residuales menos el número de hiperparámetros relativos (Harvey, 1999). La verificación se complementa con la verificación de normalidad y homocedasticidad de los residuos y la prueba de la calidad predictiva.

\subsection{Extracción de componentes no observables}

Una vez que un modelo es puesto en forma espacio estado, una herramienta importante para la estimación de los parámetros es el filtro de Kalman. Los estimadores que proporciona el filtro de Kalman son del tipo lineal, insesgado y óptimo (Peña, 2010).

Se usará el filtro de Kalman con la finalidad de calcular pronósticos mínimos cuadráticos del vector estado basado en los datos observados. En sus orígenes el filtro de Kalman proporcionaba una solución recursiva para el problema del filtrado lineal de datos discretos (Harvey, 1999). El filtro se enmarca en el modelo espacio estado bajo la suposición de que existen una o más variables o parámetros con los que se pueden describir completamente el estado en que se encuentra un sistema en un momento determinado, por ello al conjunto de variables de estado se le denomina "vector de estado" $\left(\beta_{t}\right)$, el cual no se puede observar de manera directa.

Por otro lado se tiene $y_{t}$ (vector o escalar), que constituye el vector de observación (puede ser un vector o un escalar). $Y_{t}$ puede ser una combinación lineal de las variables de estado, más un error de perturbación $\epsilon_{t}$. Por ejemplo, $\beta_{t}$ puede ser la presencia del evento Niño, el cual no se determina directamente, sino a través de sus manifestaciones de disminución en la capturadesembarque de anchoveta contaminadas además por ruido, $y_{t}$. De acuerdo a esto la ecuación de medición o de observación es: $Y_{t}=x_{t} \beta_{t}+\epsilon_{t}$. 
La matriz $x_{t}$ se supone conocida y el error de medición $\epsilon_{t}$ se distribuye como una normal con esperanza 0 y varianza $\sigma^{2}$. Se pueden incorporar variables explicativas, siendo que esta ecuación es similar al modelo lineal clásico con coeficientes variables.

Se supone que la forma como $\beta_{t}$ cambia a través del tiempo, es conocida como la ecuación de transición o del sistema $\beta_{t}=T_{t} \beta_{t-1}+d_{t}+G_{t} w_{t} ; T_{t}$, matriz de transición, $d_{t}$ vector de controles externos y la matriz $G_{t}$ son conocidos; $w_{t}$ se distribuye normalmente con esperanza cero y varianza $\sigma_{w}^{2}$ conocida. La ecuación de transición corresponde a un proceso markoviano, con coeficientes variables. La especificación de los errores puede generalizarse de forma que $\sigma_{\epsilon}^{2}$ y $\sigma_{w}^{2}$ dependen del tiempo por lo cual se agrega un subíndice $t$, con lo cual se tiene la formulación general del modelo estado espacio:

$$
\left\{\begin{array}{l}
y_{t}=x_{t} * \beta_{t}+\epsilon_{t} \\
\beta=T_{t} \beta_{t-1}+d_{t}+G_{t} w_{t}
\end{array}\right.
$$

Para la construcción del filtro de Kalman, se parte del supuesto de normalidad del vector de estado inicial y de los términos de perturbación del sistema y el método es el siguiente:

- Se formula el modelo bajo la forma espacio-estado y se considera un conjunto inicial de valores iniciales para los parámetros.

- Se generan los errores de predicción del modelo.

- Se usan estos errores para evaluar en forma recursiva la función de verosimilitud hasta maximizarla.

Usando la notación de espacio estado se tendría:

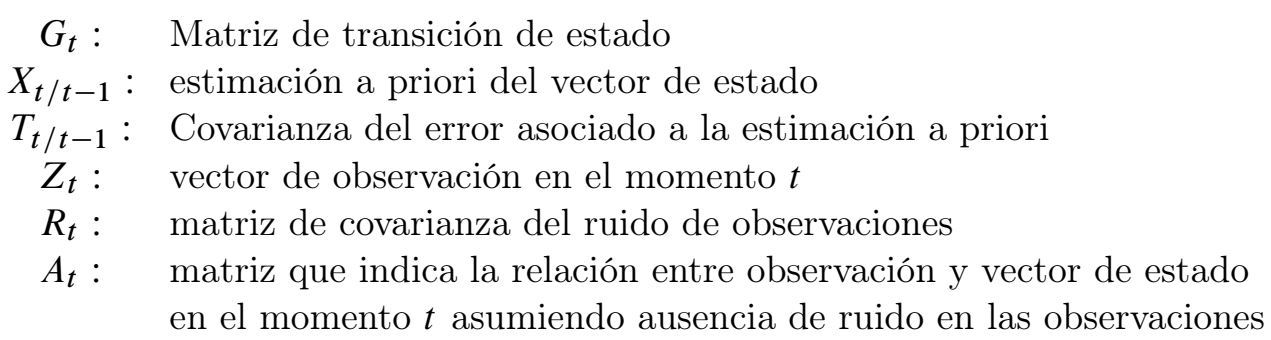

Estimación a priori: $\widehat{x}_{t / t-1}=G_{t} x_{t / t-1}$.

Covarianza del error asociada a la estimación a priori:

$$
T_{t / t+1}=G_{t} T_{t-1 / t-1} G_{t}^{T}
$$

Actualización de la medición: $\widehat{y}_{t}=Z_{t}-A_{t} \widehat{x}_{t / t-1}$.

Ganancia de Kalman: $K_{t}=T_{t / t-1} A_{t}^{T}\left(A_{t} T_{t / t-1} A_{t}^{T}+R_{t}\right)^{-1}$.

Estimación a posteriori: $\widehat{x}_{t / t}=\widehat{x}_{t / t-1}+k_{t} \widehat{y}_{t}$.

Covarianza del error asociado a la estimación a posteriori:

$$
\widehat{T}_{t / t}=\left(I-K_{t} A_{t}\right) T_{t / t-1}
$$

De esta manera se obtiene una solución óptima ya que el filtro combina toda la información observada así como el conocimiento previo acerca del comportamiento del sistema para producir 
una estimación del estado minimizando el error. El filtro recalcula la solución cada vez que una nueva observación ingresa al sistema.

Sea el vector estado $\xi_{t+1}$ condicionado al vector de observación $Y_{t}$ bajo la forma $\xi_{t+1 / t}=$ $E\left(\xi_{t+1} / Y_{t+1 t}\right)$ donde:

$$
Y_{t}=\left(\dot{y}_{t}, \dot{y}_{t-1}, \ldots, \dot{y}_{1}, \dot{x}_{t}, \dot{x}_{t-1}, \ldots, \dot{x}_{1}\right)^{\prime} \text { y } E\left(\xi_{t+1} / Y_{t+1 t}\right)
$$

La matriz de covarianzas de esta estimación de dimensión $r \times r$, se expresa como:

$$
P_{r+1 / r}=E\left(\left(\xi_{r+1}-\xi_{r+1 / r}\right)\left(\xi_{r+1}-\xi_{r+1 / r}\right)\right)^{\prime}
$$

El filtro de Kalman calcula de manera recursiva los pronósticos, generando $\widehat{\xi}_{1 / 0}, \widehat{\xi}_{2 / 0}, \ldots, \widehat{\xi}_{r / r-1}$. Se puede representar el modelo lineal general espacio estado en términos de los componentes no observables con distribución normal para los términos de perturbación de la siguiente forma:

$$
\begin{aligned}
y_{t}=Z_{t}+\alpha_{t}+\epsilon_{t} & \epsilon_{t} & \sim N\left(0, H_{t}\right) & \\
\alpha_{t+1}=T_{t} \alpha_{t}+R_{t} \eta_{t} & \alpha_{t} & \sim N\left(0, Q_{t}\right) & \eta_{t} \sim N\left(\alpha_{t}, P_{t}\right)
\end{aligned}
$$

donde $Y_{t}$ es el vector de observaciones, $\alpha_{t}$ es el vector de estado.

El siguiente paso es formar las distribuciones de ambos vectores $\alpha_{i}$ y de $y_{i}$, lo cual se realiza en forma recursiva y condicional.

Así se tiene la distribución del vector de observaciones condicionado al vector estado y vector de observaciones desfasado.

Y la distribución del vector de estado condicionado al vector de estado y vector de observaciones desfasado resulta:

$$
f\left(y_{t} \mid \alpha_{1}, \ldots, \alpha_{t}, Y_{t-1}\right)=f\left(y_{t} \mid \alpha_{t}\right) f\left(\alpha_{t+1} \mid \alpha_{1}, \ldots, \alpha_{t}, Y_{t}\right)=f\left(\alpha_{t+1} \mid \alpha_{t}\right)
$$

A partir del término de perturbación $\eta_{t}$ de la ecuación que presenta distribución normal con $E\left(\eta_{t}\right)=\alpha_{t}$ y $E\left(\eta_{t} \eta_{t-1}\right)=P_{t}$, se trata de determinar la distribución condicional

$$
f\left(\alpha_{t+1} / Y_{t}\right) \text { para } t=1, \ldots, n
$$

Siendo que esta es un subconjunto de distribuciones condicionales normales, se deduce que también se trata de una distribución normal condicionada.

De aquí que la distribució buscada será determinada conociendo su media y varianza condicionales, es decir:

$$
\alpha_{t+1}=E\left[\alpha_{t+1} \mid Y_{t}\right], P_{t+1}=E\left[\alpha_{t} \alpha_{t+1} \mid Y_{t}\right]
$$

El error de predicción $\lambda_{t}$ se determina como la diferencia entre $y_{t}$ y $\widehat{y}_{t}$ estimado, es decir de $y_{t}$ condicionado a $Y_{t-1}: \lambda_{t}=y_{t}-E\left[y_{t} \mid Y_{t-1}\right]$.

A partir de aquí se presentan los siguientes teoremas los cuales hacen posible actualizar en forma recursiva el sistema, cada vez que se incorpora una nueva observación. El filtro de Kalman para el modelo lineal general espacio estado se representa por:

$$
\begin{array}{rr}
\lambda_{t}=y_{t}-Z_{t} \alpha_{t}, & F_{t}=Z_{t} P_{t} Z_{t}^{\prime} \\
K_{t}=T_{t} P_{t} Z_{t}^{\prime} F_{t}^{-1}, & L_{t}=T_{t}-K_{t} Z_{t}, t=1, \ldots, n \\
\alpha_{t+1}=T_{t} \alpha_{t}+K_{t} \lambda_{t}, & P_{t+1}=T_{t} P_{t} L_{t}^{\prime}+R_{t} Q_{t} R_{t}^{\prime}
\end{array}
$$


$\alpha_{t}$ y $P_{t}$ son, respectivamente, la media y la matriz de varianzas del vector correspondiente al estado inicial $\alpha_{t}$.

Cuando las matrices involucradas en el modelo lineal general espacio estado no dependen del tiempo, la recursión para el cálculo de $P_{t+1}$ converge a una matriz constante denotada por $\bar{P}$, la cual es solución de la ecuación matricial:

$$
\bar{P}=T \bar{P} T^{\prime}-T \bar{P} Z^{\prime} \bar{F}^{-1} Z \bar{P} T^{\prime}+R Q R^{\prime}
$$

Donde: $\bar{F}=Z \bar{P} Z^{\prime}+H$

La solución alcanzada luego de la convergencia a $\bar{P}$, se denomina solución de estado estable del filtro de Kalman.

La utilización del estado estable después de la convergencia conduce a un ahorro considerable en cálculos, puesto que las matrices $F_{t}, K_{t}=M_{t} \bar{F}^{-1}$ y $P_{t+1}$ no necesitan ser actualizadas (Newton, H. Joseph, 1988).

En el modelo Gaussiano, el filtro de Kalman permite obtener la distribución de la media y matriz de covarianza de $\alpha_{t}$ condicionada a la información disponible en el momento $t$. Se puede generalizar que el vector de medias condicionales es el estimador mínimo cuadrático de $\alpha_{t}$. Este estimador es insesgado en el sentido que la esperanza del error de estimación es cero.

\section{Estimación Máximo Verosímil}

La función de densidad conjunta está dada por: $L(y: \psi)=\prod_{i=1}^{T} p\left(y_{i}\right), p\left(y_{i}\right)$ es la función densidad de probabilidad.

La característica principal de un modelo de una serie de tiempo es que las observaciones no son independientes. De esta forma la función de densidad conjunta y el procedimiento de máxima verosimilitud no es aplicable. En esta situación, se utiliza la función densidad de probabilidad condicional:

$$
L(y: \psi)=\prod_{i=1}^{T} p\left(\frac{y_{i}}{Y_{t-1}}\right)
$$

Donde $p\left(\frac{y_{i}}{Y_{t-1}}\right)$ denota la distribución de $y_{t}$ condicional al conjunto de observaciones en $t-1$, esto es $Y_{t-1}=y_{t-1}, y_{t-2}, \ldots, y_{t}$.

Si los disturbios y el vector de estado inicial tienen distribución normal multivariante, la distribución de $y_{t}$, condicional al $Y_{t-1}$ es normal. Además la media y matriz de covarianzas de la distribución condicional están dadas directamente por el filtro de Kalman (Harvey, 1999).

Por otro lado: Sea $\Psi$ el estimador máximo verosímil del vector $\Psi_{n \times 1}$, la matriz de información $I(\Psi)$ converge a una función densidad $I A(\Psi)$ bajo la forma:

$$
I A(\Psi)=\lim T^{-1} I(\Psi)
$$

Sujeto a ciertas condiciones de regularidad $\sqrt{T(\Psi-\psi)}$ tiene una distribución normal multivariante con vector de media cero y matriz de covarianza $I A^{-1}(\Psi)$, este resultado es extensivo para $\Psi$ asintóticamente normal con media $\psi$ y matriz de covarianza $T^{-1} I A^{-1}(\Psi)$.

Estos resultados están sujetos a las siguientes condiciones:

- $\Psi$ es un punto interior del espacio de parámetros.

- La derivada del $\log (L)$ superior al orden 3 con respecto a $\psi$ existe y es continua en la vecindad del verdadero valor del parámetro.

- $\psi$ es identificable. 


\section{Variables}

- Variable independiente: Temperatura superficial del mar peruano

- Variable dependiente: Captura-desembarque de anchoveta

\section{Hipótesis}

El orden de una modelización bivariada de la captura-desembarque de anchoveta en función de la temperatura superficial del mar permite determinar el orden de los hiperparámetros en el modelo estructural.

\section{Tipo y diseño de investigación}

La investigación es de tipo correlacional y aplicada, según Arnau Gras (Arnau Gras, 1995) los estudios de series de tiempo corresponden a los diseños de investigación longitudinales, para los cuales la determinación de la unidad de análisis, población y muestra tiene características especiales y no es frecuente su definición.

\section{Unidad de análisis}

Se tienen dos unidades de análisis:

- La biomasa de anchoveta por zona de pesca, frente a las costas del Callao.

- El mar peruano, en términos de la zona de pesca en el que se encuentra la anchoveta, frente a las costas del Callao.

\section{Población en estudio}

Biomasa de anchoveta en el mar peruano, frente a las costas del Callao. Enero de 1959 - Junio 2014.

\section{Tamaño de muestra}

En estudios de series de tiempo, cada registro en el tiempo corresponde a una muestra de tamaño uno.

\section{Selección de la muestra}

La muestra fue proporcionada por la Dirección General de Investigación de Recursos Pelágicos de IMARPE en base a los registros oficiales de captura-desembarque de anchoveta realizados por las embarcaciones que realizan actividad de pesca industrial frente a las costas de Callao en el periodo comprendido entre Enero de 1959 y Junio de 2014.

Por lo tanto se considera que los datos con los cuales se ha trabajado en la presente investigación proceden de fuentes secundarias.

\section{Resultados y Discusión}

La observación de la serie Temperatura superficial del mar $\left({ }^{\circ} \mathrm{C}\right)$ con registros mensuales evidencia un patrón cíclico, pero su regularidad es muy variable, tanto en intensidad como en periodicidad. Las intensidades altas están asociadas a la presencia del Niño y las intensidades más bajas están asociadas al friaje o Evento Niña (ver Figura1). 
El desembarque de la anchoveta aumenta en épocas de friaje y disminuye en las épocas de calentamiento (Eventos Niño). Según los registros, el descenso en la captura-desembarque de anchoveta cuando la temperatura de la superficie del mar es elevada, es tan drástico que los niveles llegan prácticamente a cero (ver Figura2).

Es así como la serie captura-desembarque de anchoveta (tnm) de registro mensual muestra la presencia de un patrón de comportamiento cíclico, el cual parece evidente, pero al mismo tiempo difícil de establecer su regularidad.

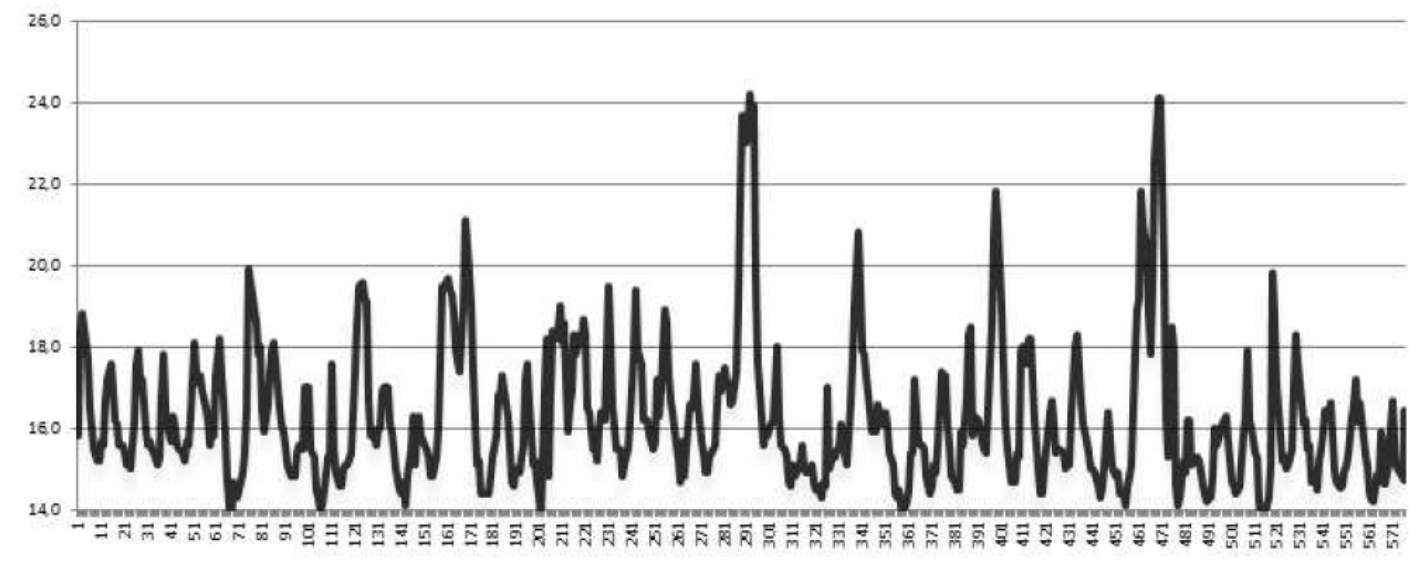

Figura 1: Temperatura ${ }^{\circ} \mathrm{C}$ superficial del Mar.Callao. Enero 1959-Diciembre 2013. Fuente: IMARPE

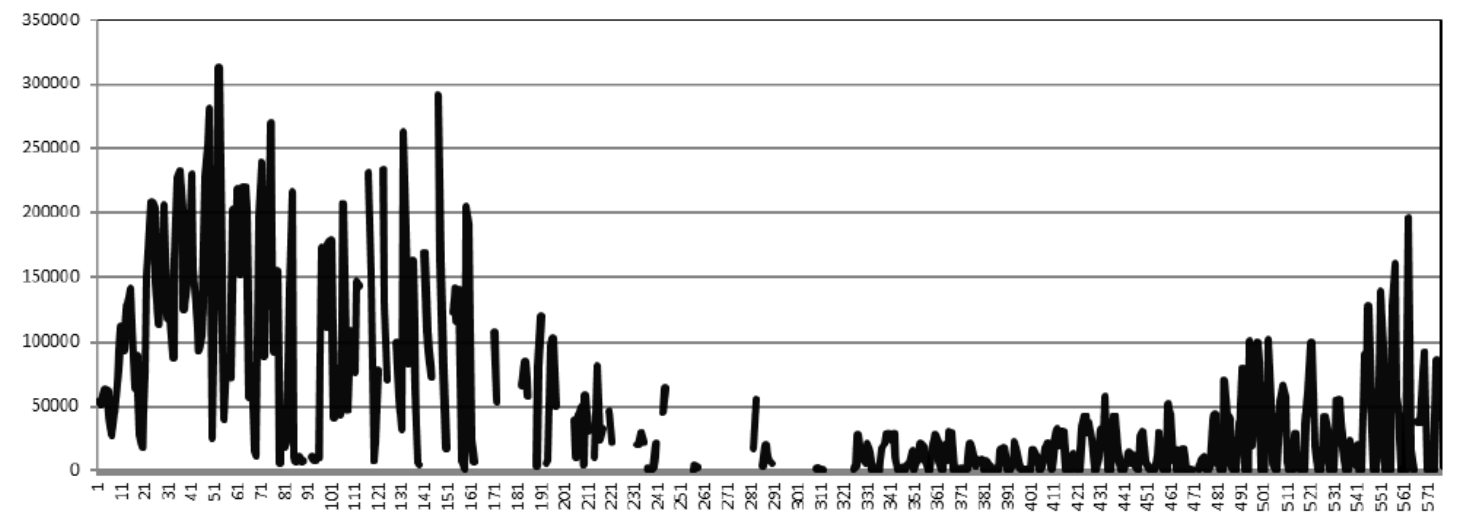

Figura 2: Captura-desembarque de Anchoveta (Tnm). Callao. Enero 1959-Diciembre 2013. Fuente: IMARPE

Por otro lado comparando el comportamiento de ambas series se intuye la relación inversa entre ambas ya que en los periodos en los cuales se registran altos niveles de temperatura, la capturadesembarque de anchoveta es menor y en los periodos con bajos niveles de temperatura la capturadesembarque aumenta.

Asumiendo que considerar las series mensuales podría incluir ruido no deseable en el análisis. Se estimaron los espectros para la serie temperatura superficial máxima anual, mediante ventana de Tuckey con punto de truncamiento 15 y amplitud de ventana 5, obteniéndose que las intensidades más altas se presentan en los periodos de 48 y 16 años (ciclos de mayor amplitud); y cada 4,8, 3,7, 5,3 años (ciclos de menor amplitud) es decir alrededor de 4 años o 48 meses. Se presenta con detalle los resultados del análisis espectral para la temperatura máxima anual (Priestley, 1981). 
Haciendo uso del espectro con la misma ventana, punto de truncamiento y amplitud, que en el caso de la temperatura máxima anual, se encuentra para la serie captura-desembarque anual de anchoveta que las intensidades más altas se presentan en los periodos de: 48 y 24 años (ciclos más largos); 9,6 y 8 años (ciclos de mediana amplitud) y cada 3,7 años (aproximadamente 4 años o 48 meses) que corresponde a los ciclos de duración más corta.

La búsqueda de periodicidades de forma univariada no permite obtener conclusiones válidas para el sistema (Box, Jenkins, \& Reinsel, 1994). En el cual la serie de entrada es la temperatura superficial y la serie de salida es la captura-desembarque de anchoveta. Se busca la asociación entre ambas series mediante los correlogramas cruzados. Estas herramientas, además nos permita identificar si la relación que se presenta entre las series es inmediata o si esta se produce de forma desfasada en el tiempo. Se han calculado las correlaciones cruzadas para ambas series bajo la forma del sistema ya mencionado bajo registros mensuales.

Con la finalidad de estabilizar las series en adelante se trabajará bajo transformación logarítmica. El resultado indica que ambas series están correlacionadas en el tiempo bajo efecto inmediato, es decir con retardo 0. La correlación cruzada indica el grado de asociación entre dos series en un momento determinado en el tiempo, y en este caso se ha encontrado que esta asociación es del 0,869 .

Cuando se calculan las correlaciones cruzadas con la temperatura máxima anual como serie de entrada y captura-desembarque promedio anual, se encuentra que la correlación cruzada más alta se presenta con un retardo 2. Tratándose de información por años, lo que en realidad se está presentado es el efecto a largo plazo de la temperatura sobre la captura-desembarque de anchoveta. Se está encontrando, en consecuencia la correlación más alta $(-0,317)$ cuando luego de 2 años, los estragos de la temperatura estarían afectando a nuevas generaciones de anchoveta. Aun cuando este resultado puede complementar muy bien los resultados obtenidos hasta el momento, no son suficientes para caracterizar las periodicidades entre ambas series. Más aún no hay registros de estudios que documenten las repercusiones en los cambios de temperaturas sobre la anchoveta después de 2 años, a menos que estas repercusiones estén en relación a otros factores adyacentes como cambios transitorios en el ecosistema que en tanto se regularizan afectan a esta especie.

Una de las bondades del análisis de series de tiempo bivariadas, es la cuantificación de la asociación de las series que conforman el sistema en cada frecuencia de importancia, es así como se presenta la coherencia espectral que es el equivalente a la función de correlación entre dos variables; la ganancia espectral, que permite determinar, como su nombre lo indica, la ganancia en el proceso de salida (captura-desembarque), en frecuencias específicas y por último el espectro de fase, que mide los saltos en el tiempo (o desfases) que se pueden presentar entre los procesos de entrada-salida.

Los periodos en los cuales se ha encontrado mayor asociación entre la Temperatura superficial mensual del Callao y la captura-desembarque mensual de Anchoveta en el Callao, corresponden a aproximadamente a la estacionalidad $(11,51)$ y a un ciclo corto de 4 años $(45,9$ meses). En el caso del ciclo la asociación es de aproximadamente $75 \%$, mientras que para la estacionalidad, la asociación está alrededor del $73 \%$. La mayor ganancia de información se consigue mediante la incorporación de la estacionalidad al modelo $(22223,591)$, esto explica por qué razón modelos como los SARIMA funcionan aceptablemente. En cuanto a la ganancia que se consigue al incorporar los ciclos esta es de 6956,519 que también resulta siendo un valor mayor a los otros registros, pero mucho menor a la ganancia estacional.

Es importante observar que al calcular el espectro de fase, este toma valores muy próximos a 0 para las periodicidades estacionales, en tanto que el espectro de fase presenta un valor de $-2,891$ para los ciclos, que implica un desfase en el que el efecto de la temperatura superficial repercute luego de 3 meses aproximadamente, sobre la biomasa estimada por la captura-desembarque (Medina Merino, 2015 [8]). 
De acuerdo a coordinaciones con el Instituto del Mar del Perú se ha confirmado que efectivamente en los últimos 5 años el periodo de vida de la anchoveta se ha alterado. En circunstancias comunes este periodo que incluye el desove y el crecimiento hasta alcanzar el tamaño adecuado de captura dura 12 meses o un año, sin embargo debido a alteraciones en el ecosistema marino asociados al incremento de la temperatura, la anchoveta ha alargado su periodo de vida en aproximadamente 3 meses en un esfuerzo de la especie por sobrevivir y multiplicarse. Esta situación estaría en concordancia con el desfase de 3 meses encontrado en el análisis que alargaría el ciclo de la anchoveta de 12 a 15 meses aproximadamente.

Un problema que es consecuencia de este desfase es que los modelos bivariados y los SARIMA de estacionalidad 12 no proporcionarían estimaciones adecuadas.

Se confirma a nivel bivariado la presencia de ciclos de aproximadamente 4 años que también se detectan en el análisis espectral univariado y cuya asociación se mide mediante la coherencia espectral. Adicionalmente se encuentran ciclos de 24 y 48 años. En todos estos la ganancia por estimar la captura-desembarque mediante la temperatura es la más alta. La coherencia espectral máxima se encuentra en los ciclos de 4 y 24 años, para 48 años se presenta una coherencia de 0,588 .

Finalmente, se verifica que a nivel de ciclos la relación entre las series es de desfase, (entre 2 y 3 años).

\section{Modelización de la influencia de la temperatura superficial sobre la captura- desembarque de anchoveta, mediante modelos estructurales en el tiempo}

Según la representación espacio-estado de las ecuaciones (1) y (2), para la modelización de la captura-desembarque mensual de anchoveta, se considera una parte estática del tipo: $Y_{t(n \times 1)}=$ $A_{(n \times k)}^{\prime} x_{t(k \times 1)}+H_{(n \times r)}^{\prime} \xi_{t(r \times 1)}+w_{t(n \times 1)}$, llamada también ecuación de medida u observación y la parte dinámica $\xi_{t(r \times 1)}=F_{r \times r} \xi_{(t-1)(r \times 1)}+v_{t(r \times 1)}$, llamada también ecuación de estado.

Se propone aplicar los parámetros del modelo bivariado para representación del sistema en el cual la entrada es la temperatura superficial mensual y la salida la captura-desembarque mensual de anchoveta.

Siendo que la transferencia del modelo bivariado SARIMA $(1,0,2)(0,1,1)_{12}$ se puede expresar como un ARMA $(13,14)$ se impondrá esta estructura al modelo espacio estado, por lo tanto el modelo dinámico de la forma:

$$
y_{t}=\phi_{1} y_{t-1}+\phi_{2} y_{t-2}+\cdots+\phi_{13} y_{t-13}+a_{t}-a_{t-1}-\cdots-\theta_{1} 4 a_{t-14}
$$

Se puede expresar en la forma espacio estado:

$$
\begin{aligned}
& y_{t}=\left[\begin{array}{llll}
1 & \theta_{1} & \cdots & \theta_{14}
\end{array}\right]\left[\begin{array}{c}
\xi_{1 t} \\
\xi_{(2 t-1)} \\
\vdots \\
\xi_{(13 t-14)}
\end{array}\right] \\
& {\left[\begin{array}{c}
\xi_{1 t} \\
\xi_{(2 t-1)} \\
\vdots \\
\xi_{(13 t-14)}
\end{array}\right]=\left[\begin{array}{cccc}
\phi_{1} & \phi_{2} & \cdots & \phi_{13} \\
1 & 0 & \cdots & 0 \\
\vdots & \vdots & \ddots & \vdots \\
0 & \cdots & 1 & 0
\end{array}\right]\left[\begin{array}{c}
\xi_{1 t-1} \\
\xi_{(2 t-2)} \\
\vdots \\
\xi_{(13 t-13)}
\end{array}\right]+\left[\begin{array}{c}
\phi_{1} \\
0 \\
\vdots \\
0
\end{array}\right] }
\end{aligned}
$$

La estimación de la ecuación de estado a partir de la información disponible hasta el momento $t=660$ y de la ecuación de medida, se realiza mediante el algoritmo del filtro de Kalman. Una primera estimación es corregida iterativamente, conforme se amplía la información disponible. 
Para un valor inicial en $t=1$ al vector $\xi$ y a su error cuadrático medio. Este no se basa en la información muestral, sino que puede considerarse como información a priori que se impone.

Con ayuda del programa Eviews (versión 8), se creó el siguiente objeto espacio que corresponde al sistema de ecuaciones estructurales en el cual la captura-desembarque de anchoveta es la serie de salida que es modelada en función de la temperatura y de variables no observables. El resultado de las ecuaciones se presenta a continuación.

$$
\begin{aligned}
& \text { Captura-desembarque }=\xi_{1 t} * \text { temperatura }+\xi_{2 t}-0,752 * \xi_{3 t}-0,395 * \xi_{4 t}- \\
& 0,565 * \xi_{5 t}+1,057 * \xi_{6 t}+0,1199 * \xi_{7 t}+0,455 * \xi_{8 t}- \\
& 1,122 * \xi_{9 t}+0,0304 * \xi_{10 t}+0,0067 * \xi_{11 t}+0,619 * \xi_{12 t}- \\
& 0,2868 * \xi_{13 t}+0,2182 * \xi_{14 t}-0,1958 * \xi_{15 t}+0,0134 * \xi_{16 t} \\
& \xi_{1 t}=c(15)+c(16) * \xi_{1 t}(-1)+\epsilon_{t} \\
& \xi_{2 t}=c(17) * \xi_{2 t}(-1)+c(18) * \xi_{3 t}(-1)+c(19) * \xi_{4 t}(-1)+c(20) * \xi_{5 t}(-1)+ \\
& c(21) * \xi_{6 t}(-1)+c(22) * \xi_{7 t}(-1)+c(23) * \xi_{8 t}(-1)+ \\
& c(24) * \xi_{9 t}(-1)+c(25) * \xi_{10 t}(-1)+c(26) * \xi_{11 t}(-1)+ \\
& c(27) * \xi_{12 t}(-1)+c(28) * \xi_{13 t}(-1)+c(29) * \xi_{14 t}(-1)+a t \\
& \xi_{3 t}=\xi_{2 t}(-1) \quad \xi_{4 t}=\xi_{3 t}(-1) \\
& \xi_{5 t}=\xi_{4 t}(-1) \quad \xi_{6 t}=\xi_{5 t}(-1) \\
& \xi_{7 t}=\xi_{6 t}(-1) \quad \xi_{8 t}=\xi_{7 t}(-1) \\
& \xi_{9 t}=\xi_{8 t}(-1) \quad \xi_{10 t}=\xi_{9 t}(-1) \\
& \xi_{11 t}=\xi_{10 t}(-1) \quad \xi_{12 t}=\xi_{11 t}(-1) \\
& \xi_{13 t}=\xi_{12 t}(-1) \quad \xi_{14 t}=\xi_{13 t}(-1) \\
& \xi_{15 t}=\xi_{14 t}(-1) \quad \xi_{16 t}=\xi_{15 t}(-1) \\
& \begin{array}{l}
\sigma_{\epsilon_{t}}^{2}=\exp (c(30))=\exp (-0,001221)=0,99878 \\
\sigma_{a_{t}}^{2}=\exp (c(31))=\exp (0,023775)=1,024
\end{array}
\end{aligned}
$$

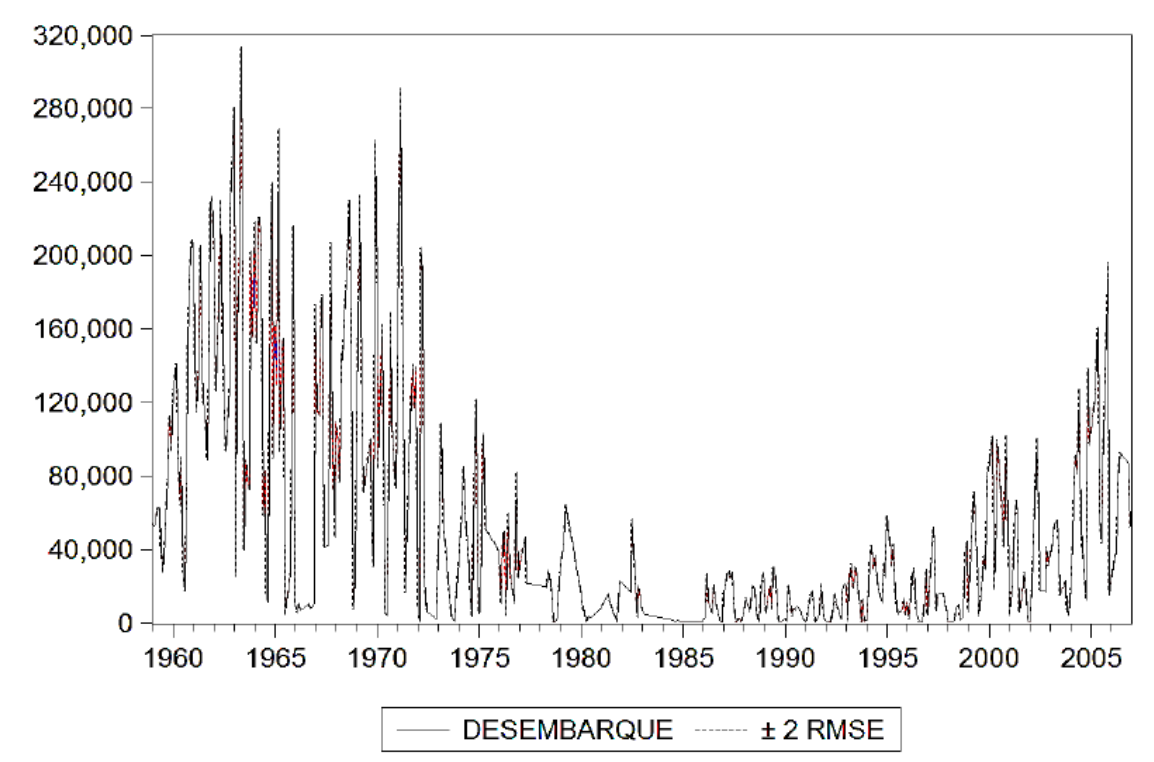

Figura 3: Simulación de la serie captura-desembarque con el modelo espacio estado superpuesto a la serie original. 
La estimación del modelo converge en la iteración 58. Todos los parámetros son significativos. Para la estimación se ha empleado el Algoritmo de Marquardt.

Se puede observar que la simulación sigue con mucha precisión el comportamiento de la serie captura-desembarque, aún mejor que en el modelo obtenido por modelización bivariada (ver Figura 3).

\section{Comprobación del Ajuste (adecuación) del Modelo}

- Prueba de Heterocedasticidad de los residuos: Bajo $H_{0}$ : La distribución de los residuos es homocedástica, al hallarse $H(m)=0,2703$ y $F(442,218,0,05)=0,827$.

- Prueba de Autocorrelación de los residuos: Bajo $H_{0}$ : Los residuos no están autocorrelacionados al hallarse para $Q=16$ hiperparámetros, $Q(16,0,05)=0,24659$, Valor crítico $\chi^{2}(135,0,05)=107,357$.

\section{Conclusiones}

1. Se estimó el comportamiento de la captura-desembarque de anchoveta en función de la temperatura superficial del mar desarrollando un modelo estructural, cuya principal característica consiste en incorporar el orden de una modelización bivariada previa para determinar el orden de los hiperparámetros del modelo estructural, logrando estimaciones muy cercanas al periodo de verificación.

2. Se identificaron patrones periódicos de la temperatura superficial del mar frente a las costas del Callao y de la captura-desembarque de anchoveta encontrando periodicidades comunes a largo plazo, que corresponden a ciclos de 48 y 4 años que pueden relacionarse con Eventos Niño de largo y corto plazo.

3. Se determinó que la temperatura superficial del mar está relacionado con la capturadesembarque de anchoveta en periodicidades variables de 12 a 15 meses, lo cual corresponde a una modificación del año vital de la anchoveta, y en aproximadamente 4 y 48 años que corresponde a los ciclos vinculados con el Evento Niño.

4. Se determinó que la temperatura superficial del mar influye en la capturadesembarque de anchoveta con un desface de 3 meses aproximadamente. De esta manera se demuestra la importancia de la temperatura superficial del mar sobre el ciclo reproductor de la anchoveta corroborando las observaciones realizadas por IMARPE en los últimos 3 años, en los cuales el ciclo de la anchoveta se ha alargado de 12 a 15 meses, en un intento de asegurar su supervivencia frente al calentamiento de las aguas superficiales del mar.

5. Mediante la aplicación de los espectros cruzados, se determinó que la ganancia máxima al modelar la captura-desembarque en función de la temperatura se producen a los 48 meses. La relación entre ambas series es del 74,5\% (coherencia espectral). En término de los efectos de la temperatura diremos que a corto plazo este es inmediato, sin embargo a largo plazo se presenta la relación con un desfase de aproximadamente 3 unidades en el tiempo.

6. Se estimó el comportamiento de la captura-desembarque de anchoveta en función de la temperatura superficial del mar desarrollando un modelo estructural, cuya principal característica consiste en incorporar el orden de una modelización bivariada previa para determinar el orden de los hiperparámetros del modelo estructural, logrando estimaciones muy cercanas al periodo de verificación. 


\section{Referencias Bibliográficas}

[1] Arnau Gras, J. (1995). Diseños longitudinales aplicados a las ciencias sociales y del comportamiento. México D.F. LIMUSA.

[2] Box, G., Jenkins, G., \& Reinsel, G., Time Series Analysis, Forecasting, New Jersey: Springer, (1994).

[3] Commandeur, J., \& Koopman, S. (2007). An Introduction to State Space Time Series Analysis. New York: Oxford.

[4] Harvey, A. C. (1999). Forecasting, structural time series models and the kalman filter. Cambridge, United Kingdom: Cambridge University Press.

[5] Hernández Sampieri, R., Fernández Collado, C., \& Baptista Lucio, P. (2010). Metodología de la Investigación. México D.F. Mc Graw Hill.

[6] IMARPE. (1 de diciembre de 2014). El Niño/La Niña Oscilaón Sur. Recuperado el 01 de diciembre de 2014, de http://www.imarpe.gob.pe/enso/Inicio/Tema1.htm

[7] Intergovernmental panel on climate change. (2007).

http://www.ipcc.ch/publications_and_data/ar4/syr/es/spms1.html.

Recuperado el 20 de Diciembre de 2011, de sitio web de IPCC Fourth Assessment Report: Climate Change 2007.

[8] Medina Merino, R. F., Modelos Estructurales - Series de Tiempo: Análisis de la Temperatura Superficial del Mar Peruano y su Influencia en la Captura Desembarque de Anchoveta, Lima: UNMSM-FCM, (2015).

[9] Peña, D. (2010). Análisis de Series Temporales. Madrid: Alianza Editorial.

[10] Pérez, C. (2006). Econometría de las Series Temporales. Madrid: Pearson Prentice Hall.

[11] Priestley, M. (1981). Spectral Analysis and Time Series. Vol 2. Multivariate Series, Prediction and Control. London: Academic Press.

[12] Ramírez, A. S. (2003). El filtro de Kalman. Banco Central de Reserva de Costa Rica, 31. 